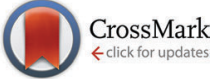

Cite this: Phys. Chem. Chem. Phys., 2016, 18, 13693

Received 13th January 2016, Accepted 8th April 2016

DOI: $10.1039 / \mathrm{c} 6 \mathrm{cp} 00259 \mathrm{e}$

www.rsc.org/pccp

\title{
Island shape and electronic structure in diindenoperylene thin films deposited on $\mathrm{Au}(110)$ single crystals $\dagger$
}

\author{
Francesca Ciccullo, ${ }^{a}$ Sabine A. Savu, ${ }^{a}$ Mathias Glaser, ${ }^{a}$ Maria Luiza M. Rocco, ${ }^{b}$ \\ Thomas Chassé ${ }^{a}$ and M. Benedetta Casu*a
}

\begin{abstract}
We have investigated diindenoperylene (DIP) thin films deposited on Au(110) single crystals, by using a multi-technique approach based on X-ray photoemission spectroscopy (XPS), resonant photoemission spectroscopy (RPES), near edge X-ray absorption fine structure (NEXAFS) spectroscopy, atomic force microscopy (AFM) and photoemission electron microscopy (PEEM). DIP molecules are physisorbed on gold, with image-charge screening playing the major role as an interface phenomenon. DIP thin films show Stranski-Krastanov growth mode and the structural herringbone arrangement mimics the arrangement found in DIP single crystals. These results are common with the (100) and (111) gold substrate geometries. On the contrary, the island aggregation is substrate geometry-dependent. This paves the way to exploit the degree of anisotropy in different lattice geometries as a tool for molecular patterning of inorganic surfaces, keeping the electronic structure preserved.
\end{abstract}

\section{Introduction}

The family of perylenes is one of the most investigated groups among organic semiconductors. Molecules with a perylene core, such as 3,4,9,10-perylene-tetracarboxylic acid dianhydride (PTCDA),$^{1-5}$ are interesting model systems for understanding growth and inorganic/organic interfaces. In addition, they are promising materials for organic electronics; for example, perylene diimide (PDI) derivatives have recently become popular in view of their use in devices, ${ }^{6-10}$ also because of their solution processability. ${ }^{11-17}$ Diindenoperylene (DIP, Fig. 1) is a perylene derivative known for forming well-organized and stable films, ${ }^{18-21}$ having very good transport properties, ${ }^{22}$ and being a good candidate as a donor material in organic photovoltaic cells. ${ }^{23}$

In previous studies thin films of DIP deposited on polycrystalline gold, $\mathrm{Au}(111)$ and $\mathrm{Au}(100)$ single crystals have been investigated elucidating the ongoing interface mechanisms and the influence of the geometry of the substrate. ${ }^{24-26}$ Here we present our results on thin films deposited on $\mathrm{Au}(110)$ single crystals, by

\footnotetext{
${ }^{a}$ Institute of Physical and Theoretical Chemistry, University of Tübingen, Auf der Morgenstelle 18, 72076 Tübingen, Germany.

E-mail: benedetta.casu@uni-tuebingen.de; Fax: +497071 29 5490; Tel: +4970712976252

${ }^{b}$ Institute of Chemistry, Federal University of Rio de Janeiro, Rio de Janeiro, 21941-909, Brazil

$\dagger$ Electronic supplementary information (ESI) available: A LEED pattern of the clean substrate. Additional thickness dependent RPES investigations. See DOI: 10.1039/c6cp00259e
}

using a multi-technique approach based on X-ray photoemission spectroscopy (XPS), resonant photoemission spectroscopy (RPES), near edge X-ray absorption fine structure (NEXAFS) spectroscopy, atomic force microscopy (AFM) and photoemission electron microscopy (PEEM). The purpose of this work is to understand the impact of the geometry of the substrate and its correlation with morphology, electronic and structural properties in the organic films.

\section{Experimental section}

A clean $\mathrm{Au}(110)$ single crystal was prepared by several cycles of $\mathrm{Ar}^{+}$ ion bombardment and annealing in UHV. The crystal surface was investigated by dark-field low energy electron microscopy (LEEM) and low energy electron diffraction (LEED) using a SPELEEM system (Elmitec, GmbH) installed at the Elettra Synchrotron Laboratory, which gave a pattern with the expected $\mathrm{Au}(110)$ reconstruction $^{27}$ (see the ESI $\dagger$ ). NEXAFS, XPS and RPES measurements were performed at the beamline UE52-PGM at BESSY II, Helmholtz-Zentrum Berlin (Berlin). This beamline was characterized by a plane grating monochromator. The photon energy ranged from 100 to $1500 \mathrm{eV}$, with an energy resolving power of $E / \Delta E=10500$ at $401 \mathrm{eV}\left(c_{\mathrm{ff}}=10,20 \mu \mathrm{m}\right.$ exit slit $)$. The main chamber (base pressure $2 \times 10^{-10}$ mbar) was equipped with a standard twin anode X-ray source, a SCIENTA R4000 electron energy analyzer, and a home-made partial electron yield detector. The thin films of DIP were grown by organic molecular beam deposition (OMBD) (evaporation rate $=3 \AA \mathrm{min}^{-1}$ ) keeping the 


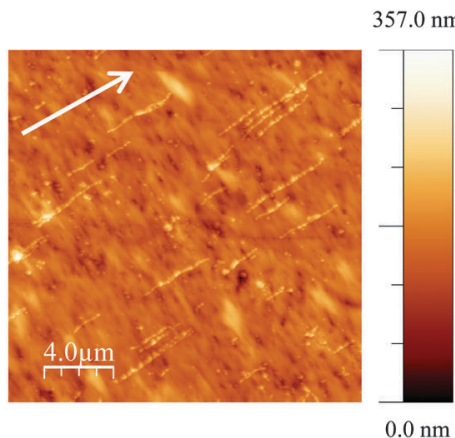

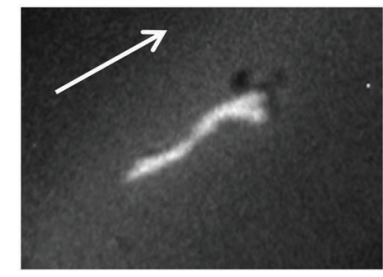
$2 \mu \mathrm{m}$

b)

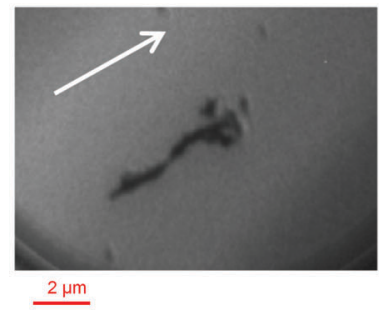

c)
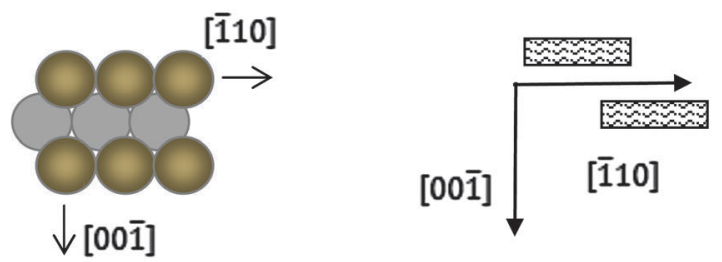

Fig. 1 Upper panel: (a) A typical $20 \mu \mathrm{m} \times 20 \mu \mathrm{m}$ AFM image of DIP films deposited on Au(110) single crystals. (b and c) XPS by PEEM imaging (field of view $10 \mu \mathrm{m} \times 10 \mu \mathrm{m}$ ), evidencing (b) in white carbon agglomeration when the photoelectron energy corresponds to the $C 1 \mathrm{~s}$ core level binding energy spectrum maximum and (c) in dark carbon-based islands when the photoelectron energy corresponds to the Au $4 f$ core level binding energy spectrum maximum. The arrow indicates the [1-10] in-plane direction. DIP molecular structure is also shown. Lower panel: Hard sphere schematic model of the fcc (110) (left), surface plane. Right: A sketch of the island aggregation as inferred from AFM and PEEM along the in-plane directions on the Au(110) surface is also shown. The islands are sketched as rectangles.

substrate at room temperature. The nominal thicknesses were measured by using the attenuation of the XPS substrate signal after DIP deposition. Details on NEXAFS normalization are given elsewhere. ${ }^{28,29}$ No degradation of the samples was observed on the time scale of all presented experiments. AFM was performed ex situ using a Digital Instruments Nanoscope III Multimode microscope in tapping mode.

\section{Results and discussion}

Fig. 1 shows the typical film morphology of DIP molecules (DIPs) on $\mathrm{Au}(110)$ single crystals. The islands form elongated agglomerates with their long axis along the [1-10] or the [001] in-plane direction on $\mathrm{Au}(110) .{ }^{26}$ The two low index surfaces in fcc lattice substrates, i.e., (100) and (110), are anisotropic. The (110) faces show atomic troughs with strong anisotropy in the atomic distances giving rise to different degrees of possible interactions across or along the troughs. ${ }^{30}$ This anisotropy, kept also after reconstruction, is the reason for the observed agglomeration of DIP islands. ${ }^{26}$ This observation of the island shape is also supported by core-level photoemission by PEEM imaging (Fig. 1). The PEEM images show bright and dark regions, evidencing in white carbon-based islands with their typical elongated shape and aggregation, when the photon energy excites photoelectrons with binding energy corresponding to the $\mathrm{C}$ 1s core level spectrum maximum (Fig. 1b), while the islands turn dark when the binding energy corresponds to photoelectrons emitted from the Au $4 \mathrm{f}$ core levels (Fig. 1c). The work function of gold is $5.2 \mathrm{eV}$. The work function decreases to $4.4 \mathrm{eV}$ within the first three DIP layers ${ }^{18}$ allowing the photoelectrons to escape and the consequent observation of a bright PEEM image. ${ }^{1,24,26}$ With increasing film thickness, this contribution is attenuated by elastic and inelastic scattering of the photoelectrons that gives rise to dark regions. ${ }^{24,28,31}$ Thus, the contrast around the island indicates the presence of a layer, confirming the Stranski-Krastanov growth mode (see Fig. 1c).

The in situ and ex situ comparison of the thin film morphology is a relevant aspect, also in view of applications. The correspondence between the results obtained in situ with PEEM and ex situ with AFM indicates that DIP films have exceptional stability against pressure gradients and that air exposure does not modify the film morphology, supporting the use of DIPs as building blocks for devices.

Note that in our previous work focused on the island shape in thin films of DIP ${ }^{26,29}$ and substituted pentacenes, ${ }^{32-34}$ we could prove that this specific elongated shape/aggregation depends exclusively on the geometry of the substrates. In particular, we could exclude that temperature, Ehrlich-Schwöbel barriers, molecular orientation, intermolecular and molecule-substrate 
interactions, and film roughness play a role under these preparation conditions. ${ }^{26}$ These results are also in good agreement with investigations performed on PTCDA, ${ }^{3,35,36}$ on needles of parahexaphenyl and sexithiophene grown on muscovite and phlogopite mica, ${ }^{37,38}$ and on nanorods of substituted pentacenes. ${ }^{32-34}$

$\mathrm{Au}(110)$ has different surface energies and different atom densities than $\mathrm{Au}(100)$ and $\mathrm{Au}(111) \cdot{ }^{30,39-41} \mathrm{~A}$ more open surface may cause a stronger bonding. ${ }^{35,42}$ XPS is a suitable technique to explore this aspect. In particular, DIP molecules are physisorbed on $\mathrm{Au}(110)$, as can be deduced from the XPS thickness-dependent investigations shown in Fig. 2 for different excitation energies (6 ^ corresponds to the completion of the first DIP monolayer $\left.{ }^{18}\right)$. The spectra do not show any change with thickness, apart from a small rigid shift towards higher binding energies $(0.1 \mathrm{eV})$, going from the thinner to the thicker films, due to the image-charge screening. ${ }^{43}$ This indicates that physisorption is the mechanism of adsorption occurring when depositing DIP on gold, independently from its lattice geometry. ${ }^{18,24,25}$

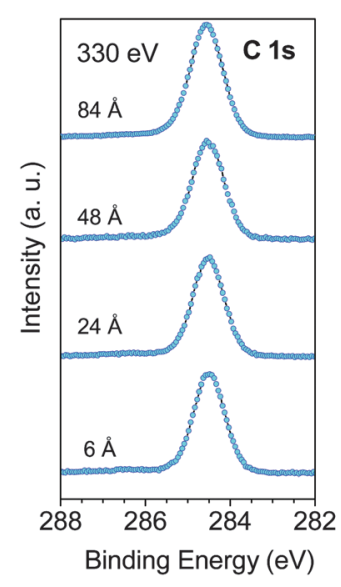

a)

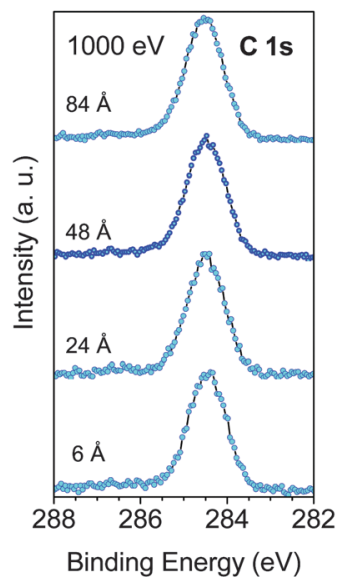

c)

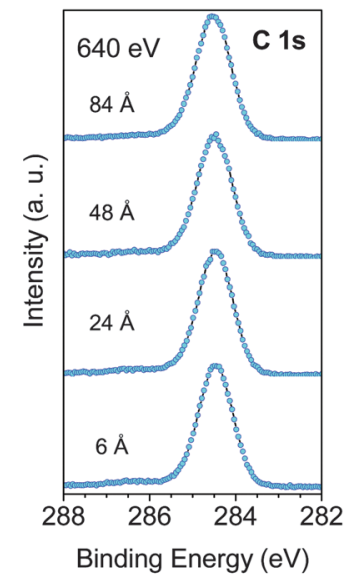

b)

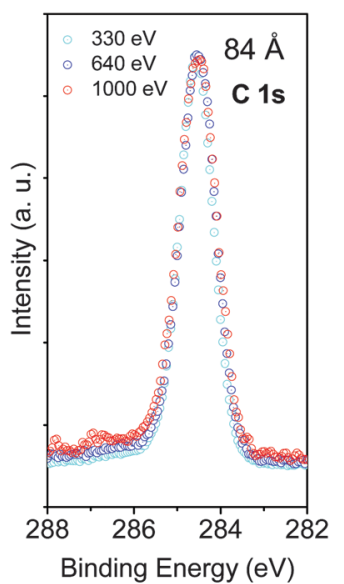

d)
Fig. $2(\mathrm{a}-\mathrm{c})$ Thickness dependent $\mathrm{C}$ 1s core level spectra recorded at 330 640, and $1000 \mathrm{eV}$ photon energy, as indicated. $6 \AA$ corresponds to the completion of the first DIP monolayer. ${ }^{18}$ (d) Spectra of the thicker film obtained for different photon energies overlapped favouring comparison. Intensities are normalized to the peak maximum.
The main line sums contributions due to photoelectrons emitted from two carbon groups: carbon atoms bonded to other carbon atoms (bridging carbon atoms) and carbon atoms bonded also to hydrogen atoms (peripheral carbon atoms). However, also among the carbon atoms belonging to each group there are further slight differences in the chemical environment. These contributions are very similar in binding energy; therefore, a fit procedure would be speculative and it will not be addressed in this work. Note that the different main line width and statistics obtained on changing the incident photon energy are due, respectively, to the different beamline resolution and the different photon flux at different photon energies.

What is important is the fact that the difference in the three experiments is due to different surface (bulk) sensitivity: higher (lower) in the case of $330 \mathrm{eV}(1000 \mathrm{eV})$. We can estimate a change of the inelastic mean free path from 4 to $15 \AA .^{44,45}$ Varying the surface sensitivity in an XPS experiment is a way to investigate the surface core level shifts (SCLS), i.e., the photoemission binding energy difference of a core level of a surface with respect to a bulk atom/molecule. We do not observe any change in the main line shape or in the binding energy of the main line within the resolution of our experiment apart from an increased shake up intensity (Fig. 2d). This result is in line with our previous experiments on PTCDA, coronene, and $\mathrm{H}_{2} \mathrm{Pc}^{44,45}$ However, we observed clear SCLS in nanorods of substituted pentacenes contacting electronegative atoms. ${ }^{32-34}$ The SCLS observed in the substituted pentacenes can be explained, as in classical semiconductors, to be due to the redistribution of the charge on the surface atoms in the ground state because of the effect of electronegativity. In fact, this model has been successfully used, for example, for SCLS in III-V semiconductors, where the levels of the group III atoms at the surface show larger binding energies while those of the group $\mathrm{V}$ atoms are shifted to smaller binding energies because of the deviation of the charge distribution at the surface in comparison with the bulk. ${ }^{46,47}$ From the comparison of the various assemblies, we could infer that there is an inter-correlation among the structural, electronic and morphological properties that leads to a different core-hole screening depending on the combination of these properties. Indeed, we observed SCLS in nanorods containing electronegative elements such as fluorine and oxygen atoms, ${ }^{32-34}$ but not in layer-by-layer films of flat lying molecules, such as PTCDA that also contains oxygen. ${ }^{44,45}$ The case of DIPs is a further tile of the mosaic that supports our interpretation: although DIP islands aggregate on $\mathrm{Au}(110)$ according to the discussed elongated shape, and structurally they are characterised by the recumbent orientation (see below), there are no observable SCLS. This clearly indicates that SCLS in rods are related to the presence of electronegative atoms, and, thus, to a morphology-enhanced deviation of the charge distribution at the surface with respect to the bulk.

NEXAFS spectra are useful not only to gain information on the unoccupied states, stemming from transitions from the 1s states to excited bound states, ${ }^{48}$ in this case, transitions from the $\mathrm{C} 1 \mathrm{~s}$ core levels to the $\pi^{*}$ and $\sigma^{*}$ molecular orbitals, ${ }^{48}$ but also to investigate the structural properties of the films. ${ }^{29,34,49,50}$ 

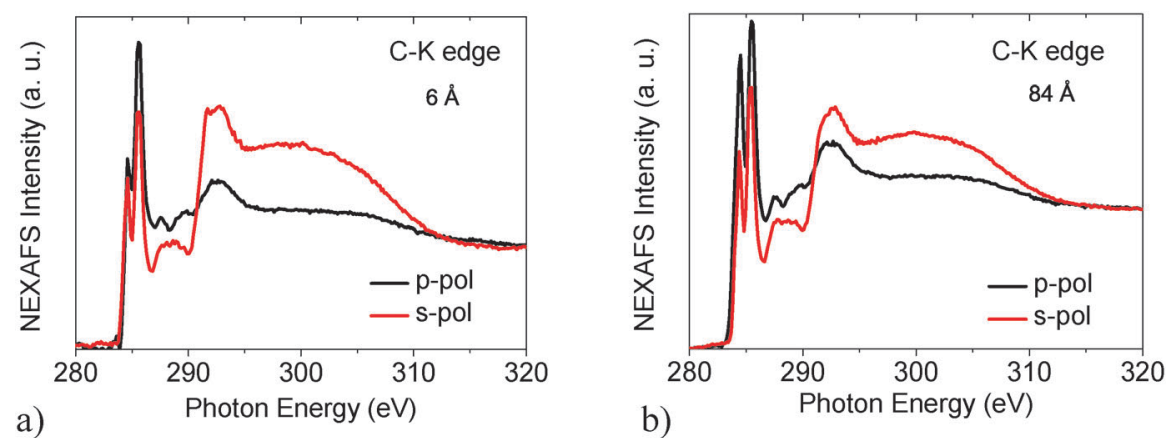

Fig. 3 C-K edges NEXAFS spectra obtained at the interface (a), compared to the multilayer (b). The spectra were taken for s-pol (red curve) and p-pol (black curve) polarization.

The NEXAFS features and their intensities are sensitive to the intermolecular interactions, ${ }^{29,34,49,50}$ and NEXAFS dichroism allows determining the orientation of the molecules with respect to the substrate..$^{48,51}$

DIP NEXAFS spectra are characterized by two main groups of resonances that dominate the spectra (Fig. 3): the $\pi^{*}$-region up to about $290 \mathrm{eV}$ and the $\sigma^{*}$-region above $290 \mathrm{eV}^{52-54}$ Looking at the feature intensities, we see that they are clearly dependent on the different polarization of the incident light. We have performed calculations of the molecular orientation by using only the $\pi^{*}$-resonance intensity. In particular, the values given in this work are calculated on the highest intensity $\pi^{*}$-resonance.

The calculated value for the angle between the substrate and the molecular plane is around $50^{\circ}$. This value could hint at two general scenarios: (1) a scenario where roughly half of the investigated domains present upright standing molecules and the remaining half flat lying molecules. (2) $50^{\circ}$ is the real molecular orientation. In standard NEXAFS the signal is averaged over the sample area spotted by the X-ray beam; thus, $50^{\circ}$ is an averaged value. Note that in the case of amorphous films or very small randomly distributed domains the spectra for the two different polarizations are expected to overlap, with no dichroism..$^{51,55,56} \mathrm{We}$ have previously investigated DIP thin films deposited on $\mathrm{Au}(111)$ single crystals by using highly laterally resolved nano-NEXAFS: ${ }^{24}$ we found that the molecules arranged themselves with a $50^{\circ}$ angle recumbent molecular orientation, according to the herringbone fashion, as seen in the bulk. ${ }^{53}$ In this case the DIP herringbone structure gives rise to contributions to both polarizations in the NEXAFS experiment. This conclusion (that is not spatially averaged because of the high lateral resolution ${ }^{24}$ ) is important for the present experiment because, comparing the nano-NEXAFS spectra ${ }^{24}$ with the spectra that we have obtained for DIPs on Au(110) crystals for the same thickness range, we note that their features are very similar, and they show the same polarization dependence. Thus, we may infer that also in this case DIPs adopt the herringbone arrangement. ${ }^{20,24}$ This is also in agreement with the arrangement, beyond the first layer, found for DIP molecules deposited on $\mathrm{Ag}(111)$ single crystals. ${ }^{20}$ Indeed, this is a consequence of the minimisation of the energy of the molecules in the solid state. If we consider two isolated molecules, the way they would structurally assemble is a compromise between the strength of the $\mathrm{C}-\mathrm{H}$ and the $\pi-\pi$ interactions. ${ }^{57}$ In a film they are not isolated: they interact with the surrounding molecules that also aim at minimising their energy because of the same interactions. In addition, the molecules feel the substrate potential. The consequence is the tendency of the molecules to assemble following the herringbone structure, with differences due to stronger/weaker substrate influence, under the same preparation conditions, that lead to a large variety of thin film polymorphisms reported in the literature..$^{57-61}$

We also observe a change with thickness in the NEXAFS intensity ratio for the two polarizations (compare Fig. 3a and b) because the molecules experience a reorientation effect, induced by their different distance from the substrate versus film thickness. This effect is quite general and is observed for other systems, such as perylene and several of its derivatives besides DIP, ${ }^{10,17,33,62}$ pentacene and its derivatives, ${ }^{34,50,61,63,64}$ and phthalocyanines. ${ }^{65-67}$

Taking the geometry of the experiment into account, and comparing the spectra obtained for different thicknesses, we observe the same features for the same polarization, and that the spectra are characterized by the same onset energy. This means that the gold/DIP interaction at the interface is weak and it does not perturb the molecular orbitals, ${ }^{68,69}$ confirming DIP physisorption. Additionally, XPS and NEXAFS do not show any fingerprint of fractional charge transfer from the substrate to the molecule. ${ }^{69}$ Contrary to what we observe in the present case, the fractional charge transfer in physisorption is characterized by unperturbed occupied states and strongly perturbed unoccupied states, accompanied by a non-rigid shift of the photoemission lines. ${ }^{69}$ Therefore, we can conclude that DIP is physisorbed on $\mathrm{Au}(110)$ single crystals with image-charge screening as the mechanism playing the major role.

Finally, considering the same thickness regime, we note that the NEXAFS features resemble very closely those found in the spectra of DIP deposited on $\mathrm{Au}(100)$ and $\mathrm{Au}(111)$ single crystals, ${ }^{25,28,29}$ indicating a similar molecular arrangement that is, therefore, not influenced by the different lattice geometries.

To further support our findings and having the opportunity to use synchrotron light as a photon source, we have performed resonant photoemission. A photoemission event leaves a core-hole behind that implies several possible relaxation effects, radiative or non-radiative, such as the Auger process. If the photoelectron is resonantly excited to a bound state like in NEXAFS, the system is neutral from the point of view of the charge. Also in this case 


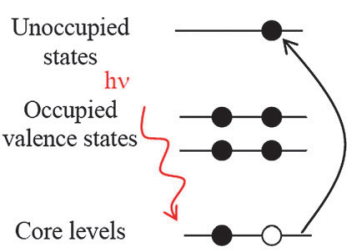

NEXAFS

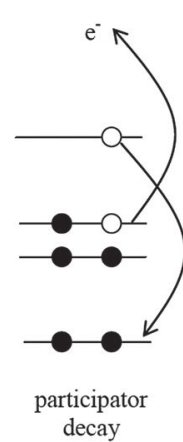

decay

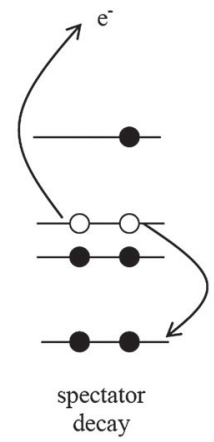

Fig. 4 Schematic view of the working principles of NEXAFS and the possible decay processes, as discussed in the text.

the de-excitation may occur via the Auger process. $^{70}$ There are two possibilities: (1) the excited electron takes part in the Auger process (participator) and the system is left with one final state hole. This is a situation energetically analogous to the photoemission case. (2) The electron remains in the normally unoccupied levels, and two valence electrons are removed (spectator), corresponding to a 2h1e (two holes one electron) final state (Fig. 4). If these phenomena occur in an organic layer close to an interface, as in this case on gold, the de-excitation is influenced by the metal/organic interface, i.e., by the substrate/ molecule interaction. In the case of chemisorption, we expect that the transfer of an electron to/from the substrate during the core-hole lifetime can influence the decay process, reducing the resonant peak intensity measured at the interface in comparison to the features obtained far from the interface. ${ }^{71-78} \mathrm{We}$ have monitored the resonant peak intensities for several film thicknesses. In particular, Fig. 5 shows the results at the interface and far from the interface (see also Fig. S3 in the ESI $\dagger$ ). We observe that in both cases (1) the spectra are characterized by a strong increase of the background at higher binding energies due to normal Auger photoelectrons. ${ }^{79,80}$ (2) The features have constant binding energy, independent of the photon energy. This behaviour indicates that their kinetic energy has a nearly linear dispersion versus the excitation energy. Therefore, the resonant enhancement of the features is due to the participator decay (see Fig. 4). The spectra recorded at the photon energy of $285.4 \mathrm{eV}$, i.e., the energy that corresponds to the most intense $\pi^{*}$ NEXAFS resonance (see Fig. 5 and compare with Fig. 3), show the biggest effect. Although the lines are quite broad, it is possible to observe that they are characterised by an increase in the intensity of the peak at $3.4 \mathrm{eV}$. This means that the resonant photoemission channel (participator) is enhanced at this energy for the interface film and the thicker film as well, and consequently no charge transfer occurs, in agreement with the results that we have obtained with XPS and NEXAFS spectroscopy. Finally, we also observe a change in the RPES line shape corresponding to the photon energy of the two highest $\pi^{*}$ NEXAFS resonances: in the photon region characteristic of the first resonance up to $284.9 \mathrm{eV}$ the line is broad and the intensity corresponding to the HOMO binding energy is enhanced, with the spectra resembling the valence band photoemission. ${ }^{18}$

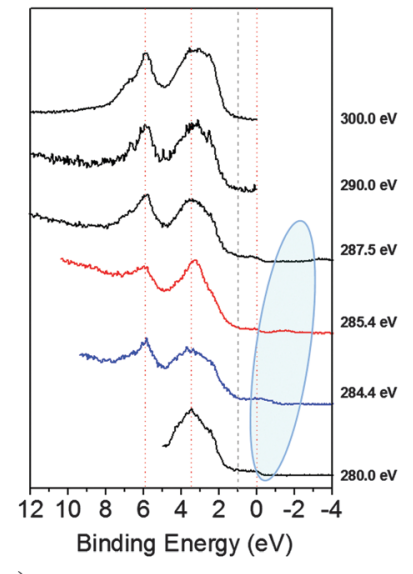

a)

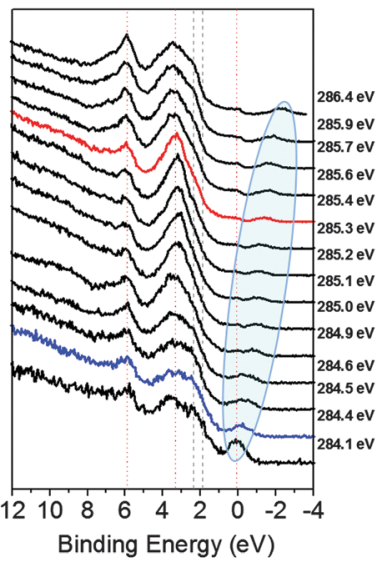

b)

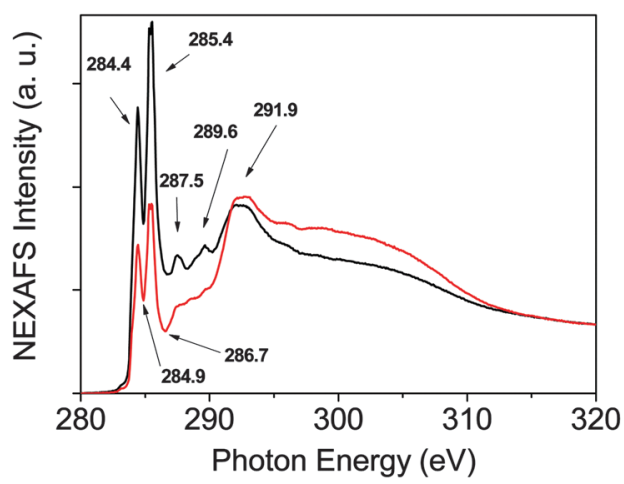

Fig. 5 Upper panel: RPES spectra measured at different photon energies, as indicated, for the (a) interface layer and (b) thicker film. The intensity is normalized to the intensity peak at $5.9 \mathrm{eV}$. The light cyan-shadowed region indicates the $C 1$ s line excited by the second order light. The HOMO and HOMO-1 binding energy positions are also indicated (grey dashed lines) according to ref. 18. Lower panel: Multilayer C-K edges NEXAFS spectra, analogous to the spectra shown in Fig. 3b. The figure shows the correspondence between the NEXAFS resonances and the RPES excitation energies.

In the region around the $\pi^{*}$ NEXAFS resonance at $285.4 \mathrm{eV}$ the line presents a characteristic pointed shape that becomes again broader above $285.7 \mathrm{eV}$. The RPES line shape is influenced by the charge-vibration coupling, ${ }^{79,81-83}$ and it reflects the dynamics of the core states. ${ }^{84,85}$ The symmetry of the spectroscopic line at $285.4 \mathrm{eV}$ indicates that the transitions involve vibrational levels having the same quantum numbers, and thus, very close energy giving rise to a narrow peak. ${ }^{79,84}$ This behaviour is common for the first layer and the multilayer, further confirming the weak interaction with the gold interface.

\section{Conclusions}

We have investigated thin films of DIP deposited on $\mathrm{Au}(110)$ single crystals focusing on the film morphology and electronic structure. To elucidate the ongoing mechanisms, we have used a multi-technique approach taking advantage of X-ray based synchrotron techniques. We have found that DIP molecules are physisorbed on gold, with image-charge screening playing the 
major role as an interface phenomenon. DIP thin films follow the Stranski-Krastanov growth mode and, structurally, they are characterised by the herringbone arrangement of the molecules, mimicking the arrangement of the molecules in the single crystal. ${ }^{53}$

Generally speaking, our work emphasizes two main aspects: the first one is related to the use of X-ray based techniques, and the second one to the island aggregation. In our previous work, we have demonstrated that the combination of thickness-dependent ultraviolet and X-ray photoemission together with X-ray absorption spectroscopy reveals the fingerprints to unambiguously describe the adsorption mechanisms at the metal/organic interface. In this work, we add RPES as a fingerprint technique. Investigating at the same time occupied (core level and valence states) and unoccupied states of a system avoids the risk that the description of the electronic structure may be influenced by the use of a specific technique, as, for example, for UPS measurements that are influenced by band dispersion or band/orbital splitting due to interaction, e.g. of two or more molecules per unit cell, or for NEXAFS measurements that convey at the same time structural and electronic information that must be decoupled to understand the ongoing phenomena. This approach reveals to be a reliable method to describe the interface phenomena, univocally identifying the electronic structure of the systems forming the interface, especially when some variations of forces leading to the interface formation might impede a clear comprehension of the bonding mechanisms, i.e. chemisorption versus physisorption.

Finally, we have found using the same preparation conditions to grow DIP films on three low index gold substrate geometries that (1) DIP is physisorbed on gold; (2) structural properties and growth mode are not influenced by the different geometry; and (3) what is straightforwardly different is the way in which islands aggregate.

If the electronic structure and the interface formation are kept identical, together with the molecular arrangement, this means that important parameters for devices such as the ionization potential are also kept constant ${ }^{63}$ and independent of the substrate geometry. This paves the way to exploit the degree of anisotropy in different lattice geometries as a tool for molecular patterning of inorganic surfaces, analogously to what is done, for example, with gold and silver nanostructures for near-optics applications, ${ }^{86}$ where the variation of geometric parameters is combined with the morphological three-dimensional control of the surface, size- and shape-tunable structures being a toolbox for nano-optics. ${ }^{86}$ Our findings could lead to the investigation of, for example, the behaviour of surface-state electrons and surface plasmons in patterned organic surfaces, towards purely organic plasmonic structures, ${ }^{87-90}$ or to the engineering of nanostructured organic-organic interfaces.

\section{Acknowledgements}

The authors thank the Helmholtz-Zentrum Berlin, Electron storage ring BESSY II, and ELETTRA Synchrotron Facility (Trieste) for providing beamtime, and Wolfgang Neu, Stephan Pohl, Matteo Lucian, and Elke Nadler for technical support.
We would like to thank Onur Mentes and Andrea Locatelli for beamtime support at ELETTRA, and helpful discussions. Financial support from the Helmholtz-Zentrum Berlin is gratefully acknowledged. MBC acknowledges financial support from DFG under the contracts CA852/5-1 and CA852/5-2. MLMR acknowledges financial support from CAPES/DAAD.

\section{References}

1 H. Marchetto, U. Groh, T. Schmidt, R. Fink, H. J. Freund and E. Umbach, Chem. Phys., 2006, 325, 178-184.

2 J. Ikonornov, O. Bauer and M. Sokolowski, Surf. Sci., 2008, 602, 2061-2068.

3 J. Ikonomov, C. H. Schmitz and M. Sokolowski, Phys. Rev. B: Condens. Matter Mater. Phys., 2010, 81, 195428.

4 L. Chkoda, M. Schneider, V. Shklover, L. Kilian, M. Sokolowski, C. Heske and E. Umbach, Chem. Phys. Lett., 2003, 371, 548-552.

5 Q. Guo, A. Paulheim, M. Sokolowski, H. Aldahhak, E. Rauls and W. G. Schmidt, J. Phys. Chem. C, 2014, 118, 29911-29918.

6 B. A. Jones, M. J. Ahrens, M.-H. Yoon, A. Facchetti, T. J. Marks and M. R. Wasielewski, Angew. Chem., Int. Ed., 2004, 43, 6363-6366.

7 R. T. Weitz, K. Amsharov, U. Zschieschang, E. B. Villas, D. K. Goswami, M. Burghard, H. Dosch, M. Jansen, K. Kern and H. Klauk, J. Am. Chem. Soc., 2008, 130, 4637-4645.

8 Y. Lin and X. Zhan, Mater. Horiz., 2014, 1, 470-488.

9 C. Musumeci, I. Salzmann, S. Bonacchi, C. Röthel, S. Duhm, N. Koch and P. Samorì, Adv. Funct. Mater., 2015, 25, 2501-2510.

10 F. Ciccullo, S. A. Savu, G. A. M. Bauer, R. Ovsyannikov, A. Cassinese, T. Chasse and M. B. Casu, Chem. - Eur. J., 2014, 21, 3766-3771.

11 J. Rivnay, L. H. Jimison, J. E. Northrup, M. F. Toney, R. Noriega, S. Lu, T. J. Marks, A. Facchetti and A. Salleo, Nat. Mater., 2009, 8, 952-958.

12 B. Yoo, T. Jung, D. Basu, A. Dodabalapur, B. A. Jones, A. Facchetti, M. R. Wasielewski and T. J. Marks, Appl. Phys. Lett., 2006, 88, 082104.

13 B. A. Jones, A. Facchetti, M. R. Wasielewski and T. J. Marks, Adv. Funct. Mater., 2008, 18, 1329-1339.

14 F. Liscio, S. Milita, C. Albonetti, P. D’Angelo, A. Guagliardi, N. Masciocchi, R. G. Della Valle, E. Venuti, A. Brillante and F. Biscarini, Adv. Funct. Mater., 2012, 22, 943-953.

15 J. Youn, G. R. Dholakia, H. Huang, J. W. Hennek, A. Facchetti and T. J. Marks, Adv. Funct. Mater., 2012, 22, 1856-1869.

16 M. Barra, F. V. D. Girolamo, F. Chiarella, M. Salluzzo, Z. Chen, A. Facchetti, L. Anderson and A. Cassinese, J. Phys. Chem. C, 2010, 114, 20387-20393.

17 F. Liscio, C. Albonetti, K. Broch, A. Shehu, S. D. Quiroga, L. Ferlauto, C. Frank, S. Kowarik, R. Nervo, A. Gerlach, S. Milita, F. Schreiber and F. Biscarini, ACS Nano, 2013, 7, 1257-1264.

18 A. Dürr, N. Koch, M. Kelsch, A. Rühm, J. Ghijsen, R. Johnson, J. J. Pireaux, J. Schwartz, F. Schreiber, H. Dosch and A. Kahn, Phys. Rev. B: Condens. Matter Mater. Phys., 2003, 68, 115428. 
19 S. Kowarik, A. Gerlach, S. Sellner, F. Schreiber, L. Cavalcanti and O. Konovalov, Phys. Rev. Lett., 2006, 96, 125504.

20 H. Huang, J.-T. Sun, Y. P. Feng, W. Chen and A. T. S. Wee, Phys. Chem. Chem. Phys., 2011, 13, 20933-20938.

21 H. Aldahhak, S. Matencio, E. Barrena, C. Ocal, W. G. Schmidt and E. Rauls, Phys. Chem. Chem. Phys., 2015, 17, 8776-8783.

22 N. Karl, Synth. Met., 2003, 133-134, 649-657.

23 J. Wagner, M. Gruber, A. Hinderhofer, A. Wilke, B. Bröker, J. Frisch, P. Amsalem, A. Vollmer, A. Opitz, N. Koch, F. Schreiber and W. Brütting, Adv. Funct. Mater., 2010, 20, 4295-4303.

24 M. B. Casu, B.-E. Schuster, I. Biswas, C. Raisch, H. Marchetto, T. Schmidt and T. Chassé, Adv. Mater., 2010, 22, 3740-3744.

25 M. B. Casu, S.-A. Savu, P. Hoffmann, B.-E. Schuster, T. O. Mentes, M. Angel Nino, A. Locatelli and T. Chassé, CrystEngComm, 2011, 13, 4139-4144.

26 M. B. Casu, S. A. Savu, B. E. Schuster, I. Biswas, C. Raisch, H. Marchetto, T. Schmidt and T. Chassé, Chem. Commun., 2012, 48, 6957-6959.

27 A. Nuber, M. Higashiguchi, F. Forster, P. Blaha, K. Shimada and F. Reinert, Phys. Rev. B: Condens. Matter Mater. Phys., 2008, 78, 195412.

28 M. B. Casu, I. Biswas, M. Nagel, P. Nagel, S. Schuppler and T. Chassé, Phys. Rev. B: Condens. Matter Mater. Phys., 2008, 78, 075310.

29 M. B. Casu, Cryst. Growth Des., 2011, 11, 3629-3635.

30 H. Brune, Surf. Sci. Rep., 1998, 31, 125-229.

31 H. Marchetto, T. Schmidt, U. Groh, F. C. Maier, P. L. Levesque, R. H. Fink, H.-J. Freund and E. Umbach, Phys. Chem. Chem. Phys., 2015, 17, 29150-29160.

32 S.-A. Savu, M. B. Casu, S. Schundelmeier, S. Abb, C. Tonshoff, H. F. Bettinger and T. Chassé, RSC Adv., 2012, 2, 5112-5118.

33 S. A. Savu, S. Abb, S. Schundelmeier, J. D. Saathoff, J. M. Stevenson, C. Toenshoff, H. F. Bettinger, P. Clancy, M. B. Casu and T. Chassé, Nano Res., 2013, 6, 449-459.

34 S.-A. Savu, A. Sonström, R. Bula, H. F. Bettinger, T. Chassé and M. B. Casu, ACS Appl. Mater. Interfaces, 2015, 7, 19774-19780.

35 J. Ikonomov, O. Bauer and M. Sokolowski, Surf. Sci., 2008, 602, 2061-2068.

36 L. Kilian, A. Hauschild, R. Temirov, S. Soubatch, A. Scholl, A. Bendounan, F. Reinert, T. L. Lee, F. S. Tautz, M. Sokolowski and E. Umbach, Phys. Rev. Lett., 2008, 100, 136103.

37 C. Simbrunner, D. Nabok, G. Hernandez-Sosa, M. Oehzelt, T. Djuric, R. Resel, L. Romaner, P. Puschnig, C. AmbroschDraxl, I. Salzmann, G. Schwabegger, I. Watzinger and H. Sitter, J. Am. Chem. Soc., 2011, 133, 3056-3062.

38 F. Balzer, M. Schiek, H.-G. Rubahn, K. Al-Shamery and A. Lützen, J. Vac. Sci. Technol., B, 2008, 26, 1619-1623.

39 J. A. Venables, Introduction to Surface and Thin Film Processes, Cambridge University Press, 2000.

40 L. Vitos, A. V. Ruban, H. L. Skriver and J. Kollár, Surf. Sci., 1998, 411, 186-202.

41 K. W. Kolasinski, Surface Science; Foundations of Catalysis and Nanoscience, Wiley, England, 2008.

42 P. Vilmercati, D. Cvetko, A. Cossaro and A. Morgante, Surf. Sci., 2009, 603, 1542-1556.
43 H. Ishii, K. Sugiyama, E. Ito and K. Seki, Adv. Mater., 1999, 11, 605-625.

44 M. B. Casu, Y. Zou, S. Kera, D. Batchelor, T. Schmidt and E. Umbach, Phys. Rev. B: Condens. Matter Mater. Phys., 2007, 76, 193311.

45 M. B. Casu, Phys. Status Solidi RRL, 2008, 2, 40-42.

46 D. E. Eastman, T. C. Chiang, P. Heimann and F. J. Himpsel, Phys. Rev. Lett., 1980, 45, 656-659.

47 R. E. Watson, J. W. Davenport, M. L. Perlman and T. K. Sham, Phys. Rev. B: Condens. Matter Mater. Phys., 1981, 24, 1791-1797.

48 J. Stöhr, NEXAFS Spectroscopy, Springer, 2003.

49 M. B. Casu, J. Electron Spectrosc. Relat. Phenom., 2015, 204, 39-48.

50 T. Breuer, M. Klues and G. Witte, J. Electron Spectrosc. Relat. Phenom., 2015, 204, 102-115.

51 J. Stöhr, K. Baberschke, R. Jaeger, R. Treichler and S. Brennan, Phys. Rev. Lett., 1981, 47, 381-384.

52 M. B. Casu, I. Biswas, B. E. Schuster, M. Nagel, P. Nagel, S. Schuppler and T. Chassé, Appl. Phys. Lett., 2008, 93, 024103.

53 M. A. Heinrich, J. Pflaum, A. K. Tripathi, W. Frey, M. L. Steigerwald and T. Siegrist, J. Phys. Chem. C, 2007, 111, 18878-18881.

54 D. G. de Oteyza, E. Barrena, M. Ruiz-Osés, I. Silanes, B. P. Doyle, J. E. Ortega, A. Arnau, H. Dosch and Y. Wakayama, J. Phys. Chem. C, 2008, 112, 7168-7172.

55 J. Stöhr and D. A. Outka, Phys. Rev. B: Condens. Matter Mater. Phys., 1987, 36, 7891-7905.

56 H. Peisert, I. Biswas, L. Zhang, M. Knupfer, M. Hanack, D. Dini, D. Batchelor and T. Chassé, Surf. Sci., 2006, 600, 4024-4029.

57 C. C. Mattheus, G. A. de Wijs, R. A. de Groot and T. T. M. Palstra, J. Am. Chem. Soc., 2003, 125, 6323-6330.

58 P. Clancy, Chem. Mater., 2011, 23, 522-543.

59 C. Ambrosch-Draxl, D. Nabok, P. Puschnig and C. Meisenbichler, New J. Phys., 2009, 11, 125010.

60 J. E. Anthony, Chem. Rev., 2006, 106, 5028-5048.

61 A. O. F. Jones, B. Chattopadhyay, Y. H. Geerts and R. Resel, Adv. Funct. Mater., 2016, 26, 2233-2255.

62 M. B. Casu, A. Scholl, K. R. Bauchspiess, D. Hubner, T. Schmidt, C. Heske and E. Umbach, J. Phys. Chem. C, 2009, 113, 10990-10996.

63 S. Duhm, G. Heimel, I. Salzmann, H. Glowatzki, R. L. Johnson, A. Vollmer, J. P. Rabe and N. Koch, Nat. Mater., 2008, 7, 326-332.

64 S. Lukas, S. Söhnchen, G. Witte and C. Wöll, ChemPhysChem, 2004, 5, 266-270.

65 I. Biswas, H. Peisert, M. B. Casu, B.-E. Schuster, P. Nagel, M. Merz, S. Schuppler and T. Chassé, Phys. Status Solidi A, 2009, 206, 2524.

66 I. Biswas, H. Peisert, M. Nagel, M. B. Casu, S. Schuppler, P. Nagel, E. Pellegrin and T. Chassé, J. Chem. Phys., 2007, 126, 174704.

67 H. Peisert, I. Biswas, M. Knupfer and T. Chassé, Phys. Status Solidi B, 2009, 246, 1529-1545.

68 M. Häming, A. Schöll, E. Umbach and F. Reinert, Phys. Rev. B: Condens. Matter Mater. Phys., 2012, 85, 1-12. 
69 S. A. Savu, G. Biddau, L. Pardini, R. Bula, H. F. Bettinger, C. Draxl, T. Chasse and M. B. Casu, J. Phys. Chem. C, 2015, 119, 12538-12544.

70 P. A. Brühwiler, O. Karis and N. Mårtensson, Rev. Mod. Phys., 2002, 74, 703-740.

71 W. Chen, L. Wang, D. C. Qi, S. Chen, X. Y. Gao and A. T. S. Wee, Appl. Phys. Lett., 2006, 88, 184102.

72 J. Schnadt, P. A. Bruhwiler, L. Patthey, J. N. O'Shea, S. Sodergren, M. Odelius, R. Ahuja, O. Karis, M. Bassler, P. Persson, H. Siegbahn, S. Lunell and N. Martensson, Nature, 2002, 418, 620-623.

73 D. G. Larrude, Y. Garcia-Basabe, F. L. Freire Junior and M. L. M. Rocco, $R S C A d v$., 2015, 5, 74189-74197.

74 F. Blobner, P. B. Coto, F. Allegretti, M. Bockstedte, O. Rubio-Pons, H. Wang, D. L. Allara, M. Zharnikov, M. Thoss and P. Feulner, J. Phys. Chem. Lett., 2012, 3, 436-440.

75 F. Petraki, H. Peisert, I. Biswas, U. Aygül, F. Latteyer, A. Vollmer and T. Chassé, J. Phys. Chem. Lett., 2010, 1, 3380-3384.

76 D. Menzel, Chem. Soc. Rev., 2008, 37, 2212-2223.

77 A. Batra, G. Kladnik, H. Vázquez, J. S. Meisner, L. Floreano, C. Nuckolls, D. Cvetko, A. Morgante and L. Venkataraman, Nat. Commun., 2012, 3, 1086.

78 T. Schiros, G. Kladnik, D. Prezzi, A. Ferretti, G. Olivieri, A. Cossaro, L. Floreano, A. Verdini, C. Schenck, M. Cox, A. A. Gorodetsky, K. Plunkett, D. Delongchamp, C. Nuckolls, A. Morgante, D. Cvetko and I. Kymissis, Adv. Energy Mater., 2013, 3, 894-902.

79 R. Friedlein, S. L. Sorensen, A. Baev, F. Gel'mukhanov, J. Birgerson, A. Crispin, M. P. de Jong, W. Osikowicz,
C. Murphy, H. Ågren and W. R. Salaneck, Phys. Rev. B: Condens. Matter Mater. Phys., 2004, 69, 125204.

80 L. Wang, W. Chen and A. T. S. Wee, Surf. Sci. Rep., 2008, 63, 465-486.

81 M. Häming, L. Weinhardt, A. Schöll and F. Reinert, Chem. Phys. Lett., 2011, 510, 82-86.

82 O. Travnikova, C. Miron, M. Bässler, R. Feifel, M. N. Piancastelli, S. L. Sorensen and S. Svensson, J. Electron Spectrosc. Relat. Phenom., 2009, 174, 100-106.

83 C. Sauer, M. Wießner, A. Schöll and F. Reinert, Phys. Rev. B: Condens. Matter Mater. Phys., 2014, 89, 075413.

84 M. Neeb, J. E. Rubensson, M. Biermann and W. Eberhardt, J. Electron Spectrosc. Relat. Phenom., 1994, 67, 261-274.

85 F. Gel'mukhanov and H. Ågren, Phys. Rep., 1999, 312, 87-330. 86 M. Fleischer, D. Zhang, K. Braun, S. Jäger, R. Ehlich, M. Häffner, C. Stanciu, J. K. H. Hörber, A. J. Meixner and D. P. Kern, Nanotechnology, 2010, 21, 065301.

87 F. J. Garcia de Abajo, J. Cordon, M. Corso, F. Schiller and J. E. Ortega, Nanoscale, 2010, 2, 717-721.

88 P. Alonso-González, A. Y. Nikitin, F. Golmar, A. Centeno, A. Pesquera, S. Vélez, J. Chen, G. Navickaite, F. Koppens, A. Zurutuza, F. Casanova, L. E. Hueso and R. Hillenbrand, Science, 2014, 344, 1369-1373.

89 D. M. Koller, A. Hohenau, H. Ditlbacher, N. Galler, F. Reil, F. R. Aussenegg, A. Leitner, E. J. W. List and J. R. Krenn, Nat. Photonics, 2008, 2, 684-687.

90 K.-J. Kim, X. Chong, P. B. Kreider, G. Ma, P. R. Ohodnicki, J. P. Baltrus, A. X. Wang and C.-H. Chang, J. Mater. Chem. C, 2015, 3, 2763-2767. 